\title{
AGILE mini-calorimeter gamma-ray burst catalog ${ }^{\star \star \star}$
}

\author{
M. Galli ${ }^{1}$, M. Marisaldi ${ }^{2}$, F. Fuschino ${ }^{2}$, C. Labanti $^{2}$, A. Argan $^{4}$, G. Barbiellini ${ }^{8,13}$, A. Bulgarelli ${ }^{2}$, P. W. Cattaneo ${ }^{7}$, \\ S. Colafrancesco ${ }^{15,16}$, E. Del Monte ${ }^{3}$, M. Feroci ${ }^{3}$, F. Gianotti ${ }^{2}$, A. Giuliani ${ }^{12}$, F. Longo ${ }^{8}$, S. Mereghetti ${ }^{12}$,

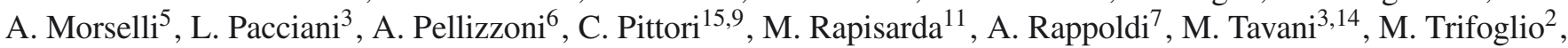
A. Trois ${ }^{6}$, S. Vercellone ${ }^{10}$, and F. Verrecchia ${ }^{15,9}$

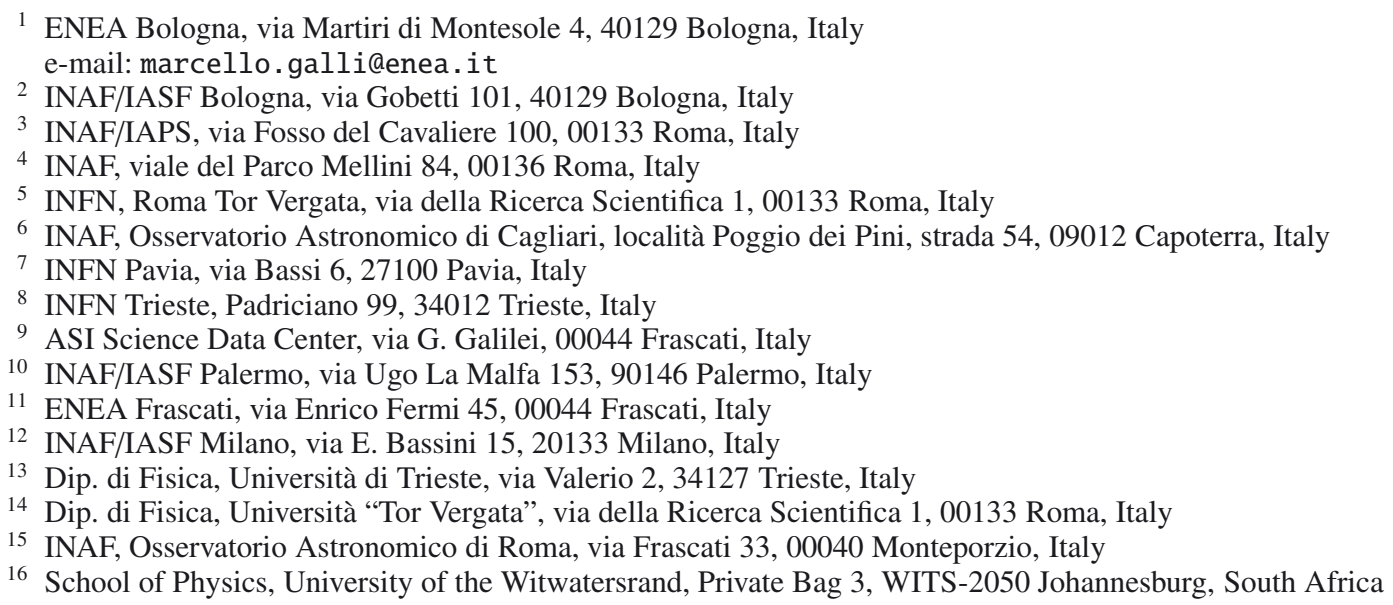

Received 3 December 2012 / Accepted 19 February 2013

\section{ABSTRACT}

\begin{abstract}
The mini-calorimeter of the AGILE satellite can observe the high-energy part of gamma-ray bursts with good timing-capability. We present the data of the 85 hard gamma-ray bursts observed by the mini-calorimeter since the launch (April 2007) until October 2009. We report the timing data for 84 and spectral data for 21 bursts.
\end{abstract}

Key words. catalogs - X-rays: bursts - gamma rays: general

\section{Introduction}

In recent years many instruments have been devoted to the study of gamma-ray bursts (GRBs), which remain one of the most puzzling phenomena in the Universe.

The energy released from these events spans a wide spectral region, from radio to $\mathrm{GeV}$, with great variations in temporal and spectral behavior (Mezaros 2006). In most cases, the emission peaks in the range 50-500 keV; for this reason most of the instruments dedicated to GRBs are optimized for detection in this energy range.

The mini-calorimeter (MCAL; Labanti et al. 2009) onboard the AGILE satellite (Tavani et al. 2009) is an instrument able to detect gamma-rays from $\sim 350 \mathrm{keV}$ to $100 \mathrm{MeV}$ region with a time resolution better than $2 \mu \mathrm{s}$. The main task of MCAL is to support the AGILE silicon tracker (Bulgarelli et al. 2010) in measuring the gamma-ray energy, but it is also used as an

* Tables 1,2 and 4 (catalog) are only available in electronic form at the CDS via anonymous ftp to cdsarc.u-strasbg. fr $(130.79 .128 .5)$ or via

http://cdsarc.u-strasbg.fr/viz-bin/qcat?J/A+A/553/A33

$\star \star$ The detailed data from the ASI Science Data Center (ASDC) is available at http://agile.asdc.asi.it/ independent transient monitor and to investigate the high-energy part of GRBs (Marisaldi et al. 2008).

Onboard the AGILE satellite the SuperAGILE (SA) instrument (Feroci et al. 2007) also acts as a GRB monitor. It is a coded mask telescope operating in the energy range $15-45 \mathrm{keV}$ with an angular resolution of 6 arcmin. Because of the very different energy ranges and the larger MCAL field of view (FOV) only a small fraction of the GRBs are detected by both instruments. The GRB data collected by SA are not homogeneous with the MCAL sample and are not considered in this paper.

Here we present a homogeneous sample of GRB data collected by MCAL between February 2008 and October 2009. During this period AGILE was operating in pointing mode, therefore a unique incoming direction in the reference frame of the spacecraft can be associated to each GRB. Since October 2009, AGILE is operating in spinning mode, due to a reactionwheel failure, scanning a large fraction of the sky with a typical angular velocity of a few degrees per second. The GRB data collected in spinning mode need different analysis methods and will be the subject of a forthcoming study.

In Sect. 2 we briefly describe the MCAL instrument, in Sect. 3 we describe the data collection and processing, Sect. 4 deals with the temporal analysis of the GRBs, Sect. 5 deals with the spectral analysis, and Sect. 6 presents our conclusions. 


\section{Instrument}

The MCAL of the AGILE satellite has been fully described elsewhere (Labanti et al. 2009): it consists of an array of $30 \mathrm{CsI}(\mathrm{Tl})$ bars, each one of size $15 \times 23 \times 375 \mathrm{~mm}^{3}$, arranged in two layers with the bars aligned along orthogonal directions for a total thickness of $3 \mathrm{~cm}$.

The scintillation light produced by the interacting gammarays is collected at each end of the bars by photo-diodes (PD); the attenuation of the light transmitted along the bar can be represented by an exponential decay; the decay coefficient has an average value of $0.028 \mathrm{~cm}^{-1}$ and has been measured for each bar before the final assembly of the instrument. The energy released in the bar and the event position along the bar are obtained according to the exponential decay law, as described in a previous paper (Labanti et al. 2009). Measurements have shown that this is a reasonable approximation and fails only for energy released at the very end of the bars, where geometrical effects dominate over attenuation.

A discriminator circuit with programmable threshold receives the sum of the electric signals from the PDs; when the sum is higher than the threshold, the PDs signals are sent to an ADC, time-tagged and stored in a circular buffer. Because of the light attenuation, this electric signal threshold does not correspond to a fixed energy threshold: the bars are more sensitive to low-energy events near the photo-diodes than at the center of the bars. The PDs are characterized by their gain $\left(\mathrm{e}^{-} / \mathrm{keV}\right)$ and the zero-level signal (offset). These parameters have been measured for each PD before launch and are used to evaluate the photon energy (Labanti et al. 2009).

The MCAL instrument is situated below the silicon tracker, but its FOV is not limited to the FOV of the tracker and acts as an all-sky monitor. The effective area of the detector is between 200 and $500 \mathrm{~cm}^{2}$, depending on photon energy and angle (Labanti et al. 2009).

A complex, fully programmable trigger logic for transient event detection is implemented on-board (Fuschino et al. 2008). This trigger logic is based on the counts given by a number of detector rate-meters (DR): four independent rate-meters are obtained by dividing each MCAL layer into two parts and summing the signals from the bars of each part. The DR counts are integrated over three energy ranges $(<1.4 \mathrm{MeV}$, 1.4-3.0 MeV, >3.0 MeV) and six time windows (1, 16, 64, 256, 1024 and $8192 \mathrm{~ms})$. These integrated counts are compared to the integrated background signal with a background integrationtime chosen among seven time intervals $(8,16,32,65,131,262$ and $524 \mathrm{~s}$ ) and delayed with respect to the DR integration times. For the 1-ms and 16-ms time windows, the MCAL is considered as a whole and not divided into four parts. An additional moving time window of $300 \mu$ s duration is implemented for the detection of very short transients.

A partial trigger signal is issued for each DR if the counts exceed a given threshold. The trigger logic compares all partial triggers; a GRB trigger signal is issued only if a proper combination of partial triggers is obtained, otherwise the event is rejected. Different combinations of DRs, thresholds, time windows, and background integration-times, can be chosen; moreover, the trigger criterion can be either static or dynamic: in the static criterion the threshold is fixed, while in the dynamic criterion the threshold is proportional to the standard deviation of the background. The GRB end time is determined by the time at which all DRs decrease below given thresholds; static and dynamic criteria are also implemented for the GRB ending time choice (TSTOP time). The trigger logic has been tuned during the first months of the mission, to optimize sensitivity and telemetry load, and minimizing the rate of false-positive triggers. The configuration implemented onboard requires that at least two partial triggers on different DR are issued at the same time for time windows larger than $16 \mathrm{~ms}$. For these time windows the dynamic criterion was set, requiring that a partial trigger is issued only when the count rate exceeds the measured background rate by at least five standard deviations. The requirement for two simultaneous partial triggers implies a rather uniform involvement of all detection planes and helps to reject local enhancements generated by, for example, noise increase. For time windows of $16 \mathrm{~ms}$ and shorter, the static trigger criterion was set, requiring at least seven, ten and ten counts in the $300 \mu \mathrm{s}, 1 \mathrm{~ms}$ and $16 \mathrm{~ms}$ time windows, respectively.

When a GRB trigger is issued, data are transmitted to the ground station on a photon-by-photon basis. Together with the GRB data, many seconds of data before and after the GRB are sent to ensure a proper estimate of the background level.

Since the end of the commissioning phase, MCAL, and SuperAGILE are part of the third InterPlanetary Network (IPN ${ }^{1}$; Hurley et al. 2004), devoted to the localizing of GRBs by means of triangulation among different spacecrafts.

\section{Data processing and selection}

AGILE is on a nearly equatorial low-Earth orbit with a period of about $90 \mathrm{~min}$; the MCAL data are sent once per orbit by the spacecraft to the ASI ground station based in Malindi, Kenya, during the ground-station contact phase. The data are processed as soon as they are received. The relevant data processing steps are energy evaluation, data selection, and photon list production.

- Gamma-ray energy release evaluation: the energy released in each bar is evaluated; most of the events deposit energy in a single bar. For multiple events, i.e., those involving more than one bar, we attribute to the event the sum of the energies released in all different bars.

- On-ground data selection: the data stream transmitted to the ground station for each GRB trigger is visually inspected, and spurious triggers due to known electronic noise issues are discarded. Data in which only very short timescale triggers (trigger on the 16-ms time window or less) are activated are also carefully inspected but, since they are mostly relevant for terrestrial gamma-ray flashes (TGF) science (Marisaldi et al. 2010), they are not included in the present work.

- Photon list production: for each accepted trigger a photon list is produced. The photon list contains the time of each detected photon, and the energy released in each bar. Many seconds of background data before and after the GRB data are included, as specified in the trigger logic configuration.

This catalog includes 76 GRBs triggered on the 64-ms timescale and longer, plus nine GRBs triggered on the 16-ms timescale. For the 64-ms sample $81 \%$ of the GRBs activate the lower energy triggers $(E<1.4 \mathrm{MeV})$ and only $17 \%$ and $2 \%$ the upper energy ones; the triggers for the four time windows of 64, 256, 1024 and $8192 \mathrm{~ms}$ represent 12, 34, 29, and $25 \%$ of the total. Triggers on timescales up to $16 \mathrm{~ms}$ where included only if the events were confirmed by at least another spacecraft belonging to the IPN. This latter criterion results in nine triggers on the $16 \mathrm{~ms}$ timescale only.

IPN web page: http://www.ssl . berkeley.edu/ipn3/ 


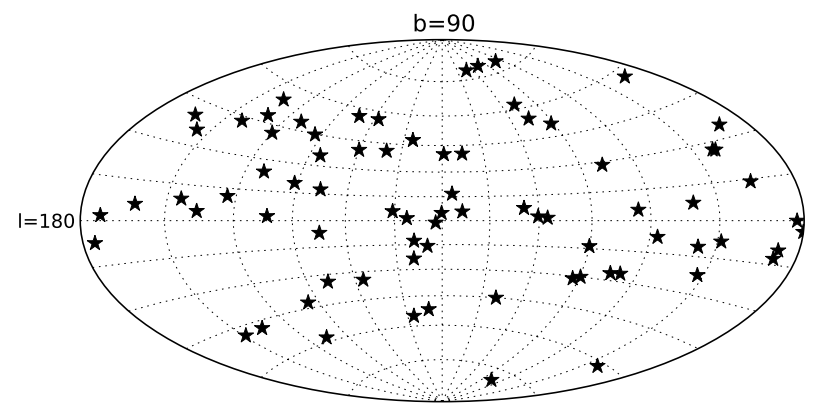

Fig. 1. Sky distribution of MCAL GRBs in galactic coordinates. The Galactic center is at the center of the figure.

The GRBs included in the catalog are listed in Table 1. The column contact is the number of the AGILE orbit in which the GRB was detected. The trigger time is the time of the GRB trigger signal (seconds from 1 January 2004); the celestial coordinates RA and Dec are given in decimal degrees and obtained from the relevant GCN circulars $^{2}$, the absence of decimals means that approximate values are obtained from the IPN data available online (Hurley et al. 2013). The column TH is the angle between the AGILE pointing direction and the direction of the GRB. The column other detections reports if the data for the GRB have been published also by other missions, or a GCN circular is issued for the detection. The GRBs for which very high energy gamma-rays have been detected by the AGILE Tracker (TR) have been highlighted.

The MCAL is an all-sky instrument, but for geometrical reasons it is more sensitive to GRBs coming from the AGILE pointing direction. In the years 2008-2009 AGILE was used to follow gamma transient pointing for many months on sources on the Galactic plane and on Galactic center. In Fig. 1 the GRB sky distribution is shown in Galactic coordinates; a greater number of GRBs can be seen on the Galactic center zone and around the Galactic plane. A list of AGILE pointings for most of the reference period is reported in (Pittori et al. 2009).

\section{Temporal analysis}

For the temporal analysis of the GRBs we computed the $T_{90}$ and $T_{50}$ time intervals, defined as the intervals containing the $90 \%$ and $50 \%$ of the total counts of gamma rays above the background. Our $T_{50}$ and $T_{90}$ measure the time width of the high energy part of the GRB (above $\sim 300 \mathrm{keV}$ ) which is seen by MCAL. We adopted the method of Koshut et al. (1996):

- the counts in the photon list are grouped in temporal bins of $0.01 \mathrm{~s}$; from which we obtained a light curve for the GRB;

- by visual analysis of the light curve, two background-only time intervals were identified, one before and one after the burst. We kept these time intervals as long as possible, to achieve a better background subtraction; for their visual identification, a binning of $0.1 \mathrm{~s}$ was sometimes used, which does not allow for an analysis of short GRBs, but gives less noisy time profiles;

- the two selected intervals were used to define a background model in the GRB time interval by a low-order interpolating polynomial (most of the time first order was sufficient);

\footnotetext{
2 GCN: A service of the Astrophysics Science Division (ASD) at NASA's GSFC: http://gcn.gsfc.nasa.gov/
}
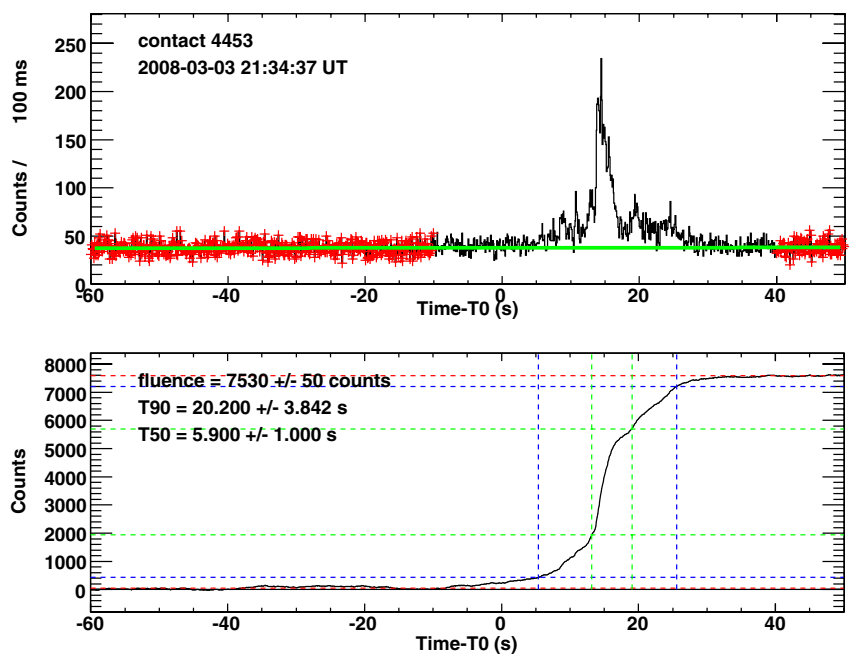

Fig. 2. GRB 080303B, time analysis. In the upper plot we show the GRB time profile; the two background zones are the time intervals: $-60 /-10$ and $40 / 50 \mathrm{~s}$. In the lower plot we show the cumulative time profile; the lines defining the fluence, $T_{50}$ and $T_{90}$ are shown as well. For a better visualization, a $0.1 \mathrm{~s}$ time binning has been used in this figure.

- the cumulative integral of the background-subtracted counts was computed for each time bin; thus we obtained a cumulative time profile;

- the mean values of the two background-only intervals in the cumulative time profile were computed. These values define a minimum and a maximum fluence level, expressed in counts. The mean value of the background region before the GRB should theoretically be zero, but in practice, as also noticed in Koshut et al. (1996), it can be different from zero, due to background fluctuations;

- a GRB fluence (in counts) is defined as the difference between the maximum and the minimum fluence level. The time interval between the points in which the cumulative time profile is $5 \%$ and $95 \%$ of the GRB fluence defines the $T_{90}$ interval. In the same way, the time points at $25 \%$ and $75 \%$ define $T_{50}$. The uncertainties in $T_{50}$ and $T_{90}$ were computed following (Koshut et al. 1996).

In Fig. 2 an example of the procedure is shown; in the upper panel we plot the time profile of GRB 080803B along with the background zones and the line fitting the background values; in the lower panel the cumulative integral is shown along with the lines defining the fluence minimum and maximum levels, the $T_{90}$ and $T_{50}$ intervals.

We performed a time analysis for 84 GRBs from our sample; GRB 080603B (contact number 5750) is confirmed by the IPN network, but too faint for a time analysis. The results are shown in Table 2. $T_{50}$ and $T_{90}$, their starting times and the boundaries of the background intervals are reported. All times are given in seconds measured from the trigger time. The column fluence contains the background-subtracted counts in the GRB region. Our $T_{50}$ and $T_{90}$ measurements refer to the high-energy part of the GRB; for this reason the duration measured by other instruments, which are sensitive to lower energies, are often longer (see Paciesas et al. 2012): we did not see the lower energy tails of some GRBs.

The main features of our sample are summarized in Table 3; our average value for $T_{90}$ is about $13 \mathrm{~s}$. We had no very long GRBs in our sample. 
Table 3. GRB temporal analysis summary.

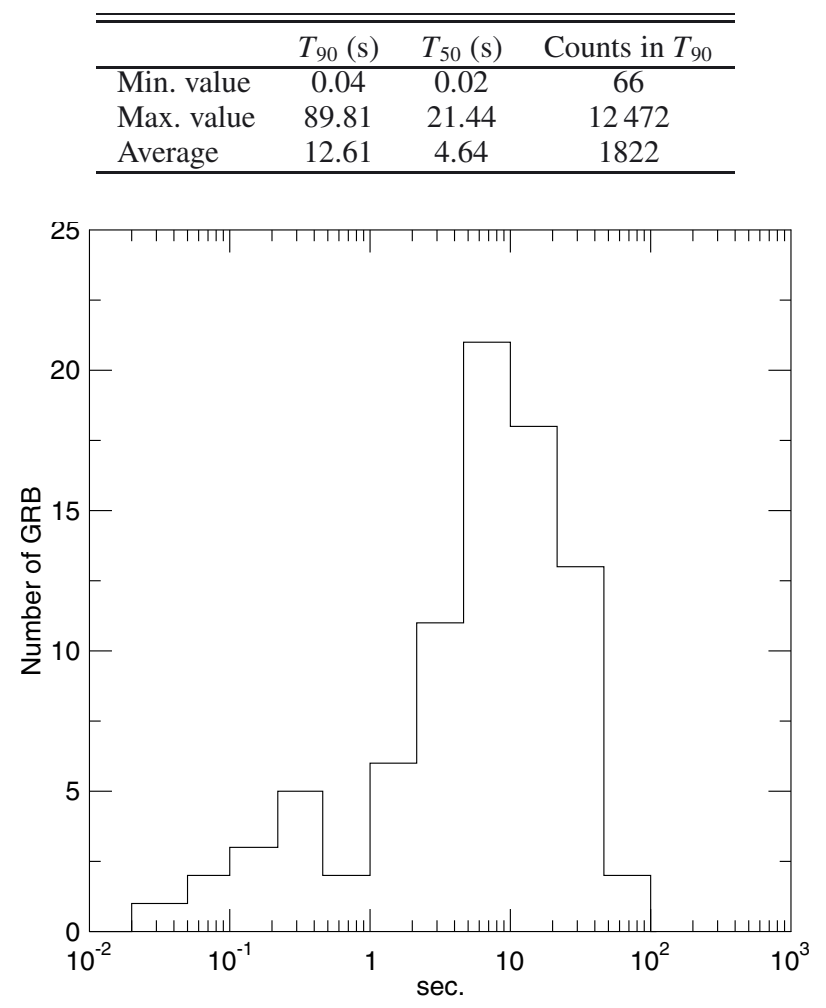

Fig. 3. $T_{90}$ distribution.

The distributions of $T_{90}$ and $T_{50}$ are shown in Figs. 3 and 4. The data are consistent with the well-known bimodal distribution (Kouveliotou et al. 1993). We see a clear peak for long GRBs and a peak for short GRBs that is less resolved due to the limited statistics for this group. The two maxima are observed at $T_{90} \sim 0.3 \mathrm{~s}$ and $T_{90} \sim 8 \mathrm{~s}$. Considering as short the GRB with $T_{90}<2 \mathrm{~s}$, we have $21 \%$ of short GRB, a value consistent with the results of instruments operating in the hard $\mathrm{X}$-ray range: Paciesas reported a value of $18 \%$ for the Fermi data (with an uncertainty of 3-4\%) and of $24 \%$ for the BATSE cata$\log$ (Paciesas et al. 2012); see also Kouveliotou et al. (1993) and Frontera et al. (2009) for previous samples.

\section{Spectral analysis}

Only a subsample of the GRBs in the catalog have enough photons to allow for a spectral analysis. Background subtraction is a critical point in the analysis, and we can obtain reliable spectra only for GRBs with more than 1000 backgroundsubtracted counts in the $T_{90}$ interval. The MCAL effective area has a cutoff below $\sim 350 \mathrm{keV}$, for this reason a fit of our data with a Band function (Band et al. 1993) is, in most cases, not suitable, since we cannot constrain the low-energy part of the spectra. We instead used a simple power law function to describe the GRB high-energy part between $400 \mathrm{keV}$ and $5 \mathrm{MeV}$ : $f(E)=N \times(E / E 0)^{-\beta}$, with a photon index $\beta$ that is similar to the $\beta$ index in the GRB Band model. We processed here all GRBs in the same way, fitting with the same simple power-law function, to obtain a set of homogeneous results. A detailed analysis for some high flux GRBs, such as GRB 090510, has been published elsewhere (Giuliani et al. 2008, 2010).

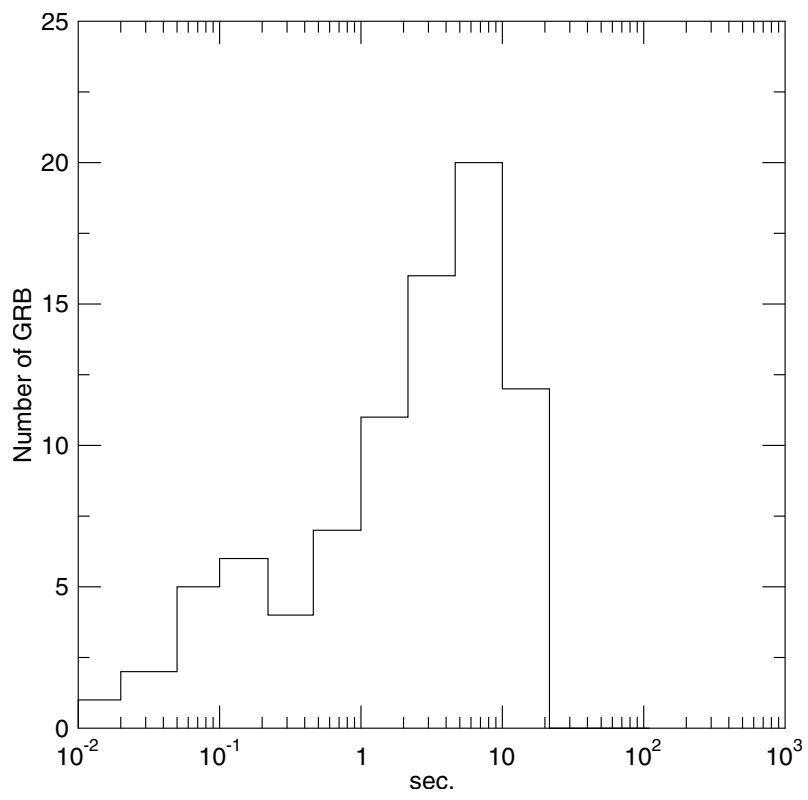

Fig. 4. $T_{50}$ distribution.

The uncertainty in the response matrix for gamma-rays coming from below the instrument is higher. These gamma-rays cross the service module of the satellite before hitting MCAL. For this reason we considered only the GRBs with a direction of less than 90 degrees from the AGILE pointing direction.

Our procedure consisted of the following steps:

- Obtaining the spectra of the detected GRB photons from the photon list. The photon list produced by the MCAL onboard software was used to obtain the spectrum of energy released in MCAL by the GRB during the $T_{90}$ time interval. A background spectrum was also extracted from the data, summing over a time interval as long as possible before the trigger (30-50 s). A large background interval smoothes the shorttime oscillations in the background, which can introduce errors when the burst fluence is low. The energies released in all bars that correspond to a single event, were summed; the bars with an energy lower than $400 \mathrm{keV}$ were discarded in this phase to minimize the contribution of possible uncertainties in diode calibrations, which mostly affect the low-energy events. The spectra were binned at a fixed bin size of $50 \mathrm{keV}$, up to $10 \mathrm{MeV}$ (200 bins). The spectra were produced in the FITS format (Wells et al. 1981).

- Dead-time correction. The anti-coincidence (AC) veto system of the AGILE tracker (Perotti et al. 2006) is active during the GRB. This system also affects the MCAL counts, blocking MCAL for $5.4 \mu \mathrm{s}$ for each AC event. With a particle background rate of $\sim 10 \mathrm{kHz}$ and a GRB photon rate of $\sim 15-30 \mathrm{kHz}$, this dead-time effect amounts to about $10-20 \%$. To account for this effect, we considered an average of the $\mathrm{AC}$ rates during the GRB and the background interval and decreased the exposure time in a consistent way.

- Fitting. The XSPEC program (Arnaud 1996) was used for fitting. We used the XSPEC CSTAT statistic, a modified version of the Cash statistic (Cash 1979), which is more appropriate when counts are low. A response matrix and auxiliary response files were computed for each GRB with the same energy binning of the spectra and considering the angle between the GRB direction and the AGILE axis. The discarding of bars with a low energy deposit was accounted for. The 
M. Galli et al.: AGILE mini-calorimeter gamma-ray burst catalog

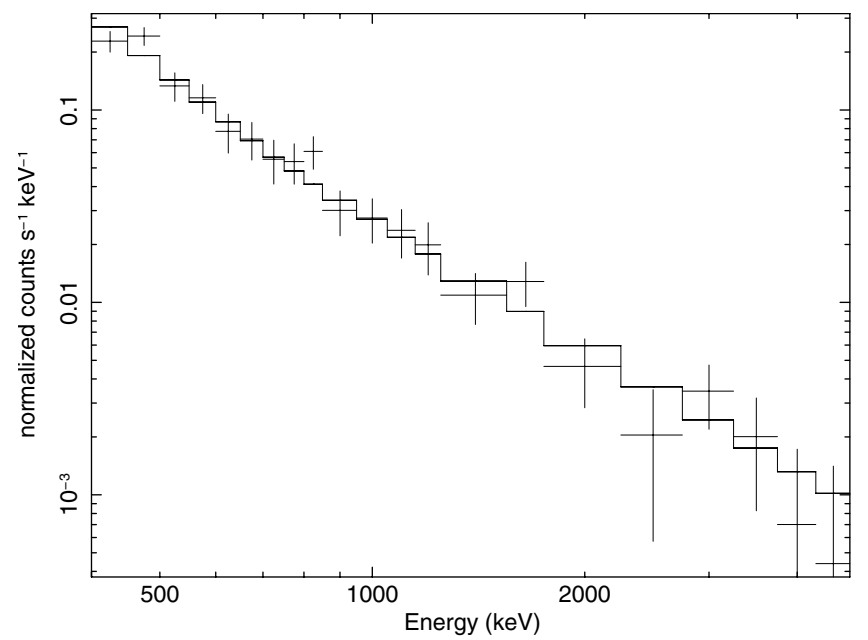

Fig. 5. GRB 080916C: spectral data and folded model from XSPEC (continuous line). The power law model is $\sim E^{-2.24}$.

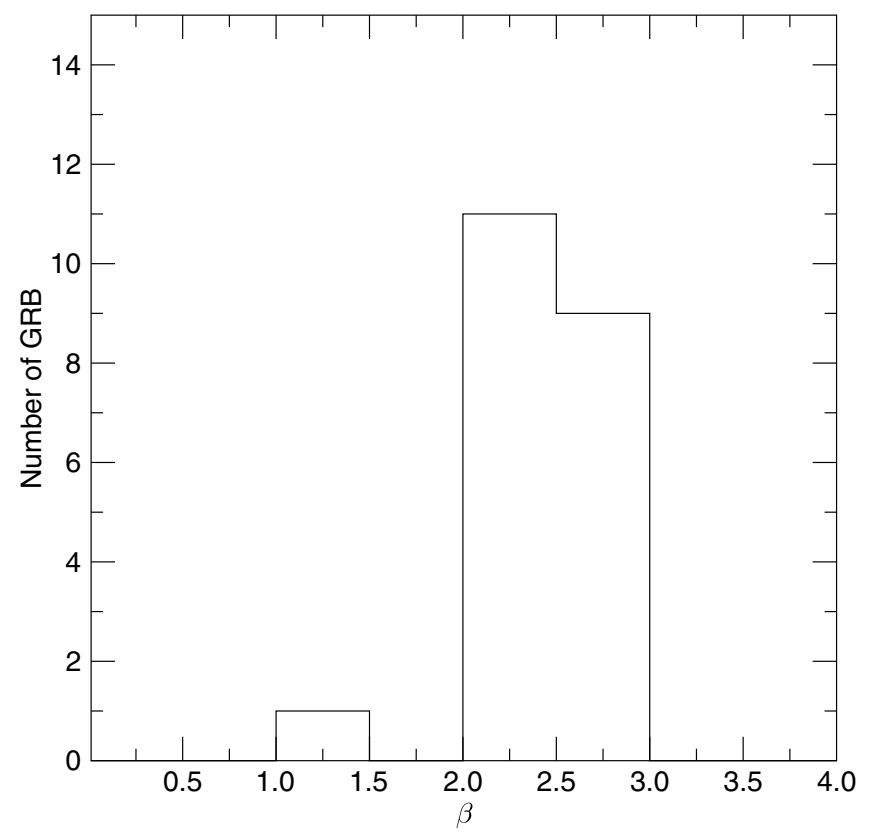

Fig. 6. Power law index $\beta$ distribution.

method used for the computation of the response matrix will be detailed in a forthcoming article.

A power law function $f(E)=N \times(E / 500 \mathrm{keV})^{-\beta}$ was used for the fits over the energy range 400-5000 keV.

In Fig. 5, an example of a GRB background-subtracted spectrum is shown along with the folded model from the XSPEC fit (continuous line); the error bars are one-sigma poisson errors. Some energy bins over $\sim 1.5 \mathrm{MeV}$ were merged.

Spectral data are shown in Table 4 , where the spectral index $\beta$ and the normalization parameter $N$ are reported along with the model flux integrated over the energy interval 400-5000 keV and averaged over $T_{90}$. We also report the fluence, integrated over $T_{90}$, the CSTAT value, and the number of degrees of freedom (d.o.f.) from the XSPEC fit.

In Fig. 6 we show the distribution of the power law index $\beta$. Most of the values are between 2 and 3. A similar distribution is found for the Band model $\beta$ parameter in the Fermi sample of Bissaldi (Bissaldi et al. 2011) or in the Fermi spectral catalog (Goldstein et al. 2012), but we see a somewhat greater number

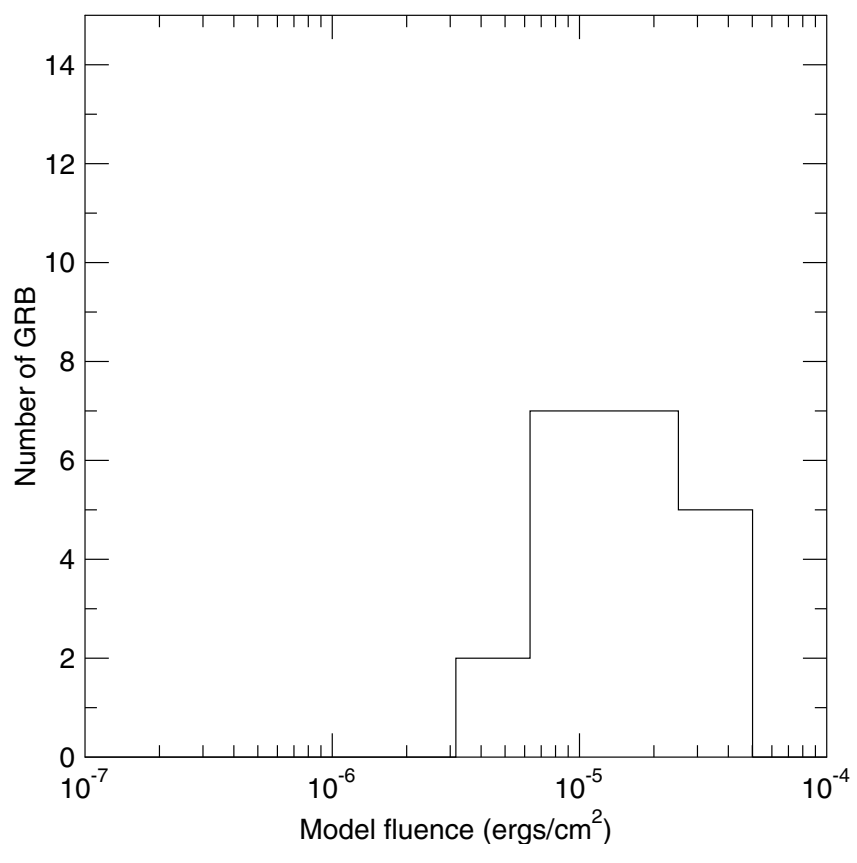

Fig. 7. Fluence distribution.

of high $\beta$ GRBs. We are still investigating this effect; a detailed comparison of our spectral data with other samples will be presented in a forthcoming paper. The model fluence distribution is shown in Fig. 7.

\section{Conclusions}

We presented the GRB data collected with the AGILE MCAL instrument between February 2008 and October 2009. The effective area of MCAL decreases below $\sim 350 \mathrm{keV}$, and this GRB sample consists of hard events, with data up to the $\mathrm{MeV}$ range. Our temporal analysis shows a $T_{90}$ distribution consistent with other studies, in spite of our higher energy range. We also presented a spectral analysis for a subsample of our data: we fitted our data with a simple power law. Our spectral index is comparable to the $\beta$ parameter in the classical band GRB model. Our results are similar to other studies, but with somewhat lower spectral indexes.

Acknowledgements. AGILE is a mission of the Italian Space Agency (ASI), with co-participation of INAF (Istituto Nazionale di Astrofisica) and INFN (Istituto Nazionale di Fisica Nucleare). This work was carried out in the frame of the ASI-INAF agreement I/028/12/0. Alessandro Ursi participated to a first step of the data analysis.

\section{References}

Aptekar, R. L., Frederiks D. D., Golenetskii S. V., et al. 1995, Space Sci. Rev., 71,265

Arnaud, K. A. 1996, Astronomical Data Analysis Software and Systems V, eds. G. Jacoby, \& J. Barnes, ASP Conf. Ser., 101, 17

Band, D., Matteson, J., Ford, L., et al. 1993, ApJ, 413, 281

Bissaldi, E., von Kienlin, A., Kouveliotou, C., et al. 2011, ApJ, 733, 97

Bulgarelli, A., Argan, A., Barbiellini, G., et al. 2010, Nucl. Instr. Meth. A, 614, 213

Cash, W. 1979, ApJ, 228, 939

Feroci, M., Costa, E., Soffitta, E., et al. 2007, Nucl. Instr. Meth. A, 581, 728

Frontera, F., Guidorzi, C., Montanari, E., et al. 2009, ApJS, 180, 192

Fuschino, F., Labanti, C., Galli, M., et al. 2008, Nucl. Instr. Meth. A, 588,17

Gehrels, N., Chincarini, G., Giommi P., et al. 2004, ApJ, 611, 1005

Giuliani, A., Mereghetti, S., Fornari, F., et al. 2008, A\&A, 491, L25 
A\&A 553, A33 (2013)

Giuliani, A., Cutini, S., Pittori, C., et al. 2009, GRB Coordinates Network, Circular 9075

Giuliani, A., Fuschino, F., Vianello, G., et al. 2010, ApJ, 708, L84

Goldstein, A., Burgess, J. M., Briggs, M. S., et al. 2012 ApJS, 199, 19

Hurley, K., Rau, A., von Kienlin, A., et al. 2004, in 5th INTEGRAL Workshop on the INTEGRAL Universe, eds. V. Schoenfelder, G. Lichti, \& C. Winkler, ESA SP, 552, 645

Hurley, K., Palshin, V. D., Aptekar, R. L., et al. 2013 [arXiv: 1301.3522]

Koshut, T. M., Precias, W. S., Kouveliotou, C., et al. 1996, ApJ, 463, 570

Kouveliotou, C., Meegan, C. A., Fishman, G. J., et al. 1993, ApJ, 413, L101

Labanti, C., Marisaldi, M., Fuschino, F., et al. 2009, Nucl. Instr. Meth. A, 598, 470

Lin, R. P., Dennis, B. R., Hurford, G. J., et al. 2002, Sol. Phys., 210, 3

Marisaldi, M., Labanti, C., Fuschino, F., et al. 2008, A\&A, 490, 1151

Marisaldi, M., Fuschino, F., Labanti, C., et al. 2010, JGR, 115, A00E13
Meegan, C., Lichti, G., Bhat, P. N., et al. 2009, ApJ, 702, 791

Mézáros, P. 2006, Rep. Prog. Phys., 69, 2259

Michelson, P. F., Atwood, W. B., \& Ritz, S. 2010, Rep. Prog. Phys. 73, 074901

Mitsuda, K., Bautz, M., Inoue, H., et al. 2007, Publ. Astron. Soc. Japan, 59, 1

Moretti, E., Longo, F., Barbiellini, G., et al. 2009, GRB Coordinates Network, Circular 9069

Paciesas, W. S., Megegan, C. A., Pendleton G. N., et al. 1999, ApJS, 122, 465 Paciesas, W. S., Megegan, C. A., von Kienlin, A., et al. 2012, ApJS, 19918

Perotti, F., Fiorini, M., Incorvaia, S., et al. 2006, NIM A, 556, 228

Pittori, C., Verrecchia, F., Chen. A. W., et al. 2009, A\&A, 506, 1563

Prest, M., Barbiellini, G., Bordignon, G., et al. 2003, Nucl. Instr. Meth. A, 501, 280

Tavani, M., Barbiellini, G., Argan, A., et al. 2009, A\&A, 502, 995

Wells, D. C., Greisen, E. W., \& Harten, R. H. 1981, A\&AS, 44, 363

Winkler, C., Courvoisier, T., Di Cocco, G., et al. 2003, A\&A, 411, L1 